\title{
Fourier Truncation Method for Fractional Numerical Differentiation
}

\author{
Ailin Qian, Jianfeng Mao \\ Department of Mathematics and Statistics, Xianning University, Xianning, China \\ E-mail: junren751113@126.com \\ Received February 13, 2011; revised May 31, 2011; accepted June 3, 2011
}

\begin{abstract}
We consider an ill-posed problem-fractional numerical differentiation with a new method. We propose Fourier truncation method to compute fractional numerical derivatives. A Hölder-type stability estimate is obtained. A numerical implementation is described. Numerical examples show that the proposed method is effective and stable.
\end{abstract}

Keywords: Inverse Problems, Ill-Posed Problem, Fractional Numerical Differentiation, Fourier Regularization, Error Estimate

\section{Introduction}

In this paper we shall consider the problem of stably calculating the fractional derivative of a function $f$ given in $L_{p}(R)$,

$$
D^{\alpha} f(x)=\frac{1}{\Gamma(1-\alpha)} \frac{\mathrm{d}}{\mathrm{d} x} \int_{-\infty}^{x} \frac{f(t) \mathrm{d} t}{(x-t)^{\alpha}},
$$

for $\alpha \in(0,1)$. Such problem is frequently encountered in many practical contexts [1-3]. It is well known that $D^{\alpha} f(x)$ is a formal solution of the Abel integral equation.

$$
\begin{aligned}
& I^{\alpha} u(x)=\frac{1}{\Gamma(\alpha)} \int_{-\infty}^{x} \frac{u(t)}{(x-t)^{1-\alpha}} \mathrm{d} t=f(x), \\
& -\infty<x<\infty .
\end{aligned}
$$

That is very important in various areas of mechanics, spectroscopy, computerized tomography [2]. The process of numerical fractional differentiation is well known to be an ill-posed problem [1-3], and it has been discussed by many authors, and a large number of different solution methods have been proposed. For references we refer the reader to [1-5] and references therein. Finite difference approaches for numerical differentiation have been used, for example, in [6-9]. However, these approaches require knowledge of a bound of the second or third derivatives of the function under consideration that are not always available. Furthermore, there exist infinitely many functions that do not have bounded sec- ond derivatives at all. The same situation occurs also in numerical fractional differentiation [2]; one requires a high smoothness of the functions under consideration that does not always exist. In the present paper, as an alternative way of dealing with fractional numerical differentiation, we introduce a new regularization method, i.e., Fourier truncation. We simply analyze the cause of illposedness of fractional numerical differentiation and then propose the method. The idea of Fourier truncation method is very simple and natural: since the ill-posedness of fractional numerical differentiation is caused by the high frequency components, we cut off them. Actually, such a similar idea of solving numerical differentiation has occurred in $[10,11]$. We can easily find this fact by studying $[10,11]$ in frequency space. However, Fourier truncation method is more direct, natural and simple.

\section{Regularization in the Fourier Domain}

In this section we simply analyze the ill-posedness of fractional numerical differentiation and discuss how to stabilize the numerical derivaties. We set a function $f(x) \in H^{p}(R)$ which is a Soblev space, $p>\alpha$. Let $f$ be the Fourier transform of $f$, i.e.,

$$
\hat{f}(\xi)=\frac{1}{\sqrt{2 \pi}} \int_{-\infty}^{+\infty} f(x) e^{-i \xi x} \mathrm{~d} x
$$

Now we consider the $\alpha$-th order derivative of function $f$. Taking the Fourier transform, we have 


$$
\left(f^{(\alpha)}(x)\right)^{\wedge}=(i \xi)^{\alpha} \hat{f}(\xi)
$$

i.e.,

$$
f^{(\alpha)}(x)=\frac{1}{\sqrt{2 \pi}} \int_{-\infty}^{+\infty}(i \xi)^{\alpha} \hat{f}(\xi) e^{i \xi x} \mathrm{~d} \xi .
$$

From the right hand side of (2.2) or (2.3), we know that $|i \xi|^{\alpha}=|\xi|^{\alpha}$ can be seen as an amplification factor of $\hat{f}(\xi)$. Therefore, when we consider our problem in $L^{2}(R)$, the exact data function $\hat{f}(\xi)$ must decay rapidly as $\xi \rightarrow \infty$. But in the applications the input data $f(x)$ can only be measured and never be exact. We assume, say that, the measured data function $f_{\delta}(x) \in L^{2}(R)$ satisfies

$$
\left\|f_{\delta}-f\right\| \leq \delta
$$

where $\|\cdot\|$ denotes $L^{2}-$ norm, the constant $\delta>0$ represents a noise level. Thus, if we try to obtain fractional numerical derivatives, high frequency components in the error are magnified and can destroy the solution. In this sense it is impossible to solve the problem using classical numerical methods and requires special techniques to be employed. In the following, we will propose our regularization strategy to deal with the ill-posed problem. However, before doing that, we impose a priori bound on the input data (this is necessary in solving ill-posed problems), i.e.,

$$
\|f\|_{p} \leq E, p>\alpha
$$

where $E>0$ is a constant, $\|\cdot\|_{p}$ denotes the norm in Soblev space $H^{p}(R)$ defined by

$$
\|f(\cdot)\|_{p}:=\left(\int_{-\infty}^{+\infty}\left(1+\xi^{2}\right)^{p}|\hat{f}(\xi)|^{2} \mathrm{~d} \xi\right)^{\frac{1}{2}}
$$

Note that (2.2) or (2.3), since the ill-posedness of the problem is caused by the high frequency components, a natural way to stabilize the problem is to eliminate all high frequencies and instead consider (2.3) only for $|\xi| \leq \xi_{\max }$, where $\xi_{\max }$ will be seen that it exists as a regularization parameter. Then we get a regularized fractional derivative

$$
R\left(f_{\delta}^{(\alpha)}(x)\right):=\frac{1}{\sqrt{2 \pi}} \int_{-\infty}^{+\infty}(i \xi)^{\alpha} \hat{f}_{\delta}(\xi) \chi_{\max } e^{i \xi x} \mathrm{~d} \xi .
$$

where $\chi_{\max }$ is the characteristic function of the interval $\left[-\chi_{\max }, \chi_{\max }\right]$, i.e.,

$$
\chi_{\max }(\xi)=\left\{\begin{array}{l}
1,-\xi_{\max } \leq \xi \leq \xi_{\max }, \\
0,|\xi|>\xi_{\max }
\end{array}\right.
$$

In the following sections we will derive an error estimate for the approximate derivative (2.7) and discuss how to compute it numerically.

\section{Error Estimate}

In this section, we derive a bound on the difference between the derivatives (2.3) and (2.7). We assume that we have an a priori bound on the exact input data. $\|f\|_{p} \leq E$ (See (2.5)). The relation between any two regularized solutions (2.7) is given by the following lemma.

Lemma 3.1. Suppose that we have two regularized derivatives $R\left(f_{1}^{(\alpha)}(x)\right)$ and $R\left(f_{2}^{(\alpha)}(x)\right)$ defined by (2.7) with data $f_{1}$ and $f_{2}$, satisfying $\left\|f_{1}-f_{2}\right\| \leq \delta$. If we select $\xi_{\max }=\left(\frac{E}{\delta}\right)^{\frac{1}{p}}, \alpha<p \leq 1$, then we get the error bound

$$
\left\|R\left(f_{1}^{(\alpha)}(\cdot)\right)-R\left(f_{2}^{(\alpha)}(\cdot)\right)\right\| \leq E^{\frac{\alpha}{p}} \delta^{1-\frac{\alpha}{p}} .
$$

Proof. From the Parseval relation we get

$$
\begin{aligned}
& \left\|R\left(f_{1}^{(\alpha)}(\cdot)\right)-R\left(f_{2}^{(\alpha)}(\cdot)\right)\right\|^{2} \\
& =\left\|R \widehat{\left(f_{1}^{(\alpha)}(\cdot)\right)}-R \widehat{\left(f_{2}^{(\alpha)}(\cdot)\right)}\right\|^{2} \\
& =\int_{-\xi_{\max }}^{\xi_{\max }}\left|(i \xi)^{\alpha}\left(\widehat{f}_{1}(\xi)-\widehat{f}_{2}(\xi)\right)\right|^{2} \mathrm{~d} \xi \\
& =\int_{-\xi_{\max }}^{\xi_{\max }}|\xi|^{2 \alpha}\left|\widehat{f}_{1}(\xi)-\widehat{f}_{2}(\xi)\right|^{2} \mathrm{~d} \xi \\
& \leq \xi_{\text {max }}^{2 \alpha}\left\|\widehat{f}_{1}-\widehat{f}_{2}\right\|^{2}=\xi_{\text {max }}^{2 \alpha}\left\|f_{1}-f_{2}\right\|^{2}
\end{aligned}
$$

Using $\xi_{\max }=\left(\frac{E}{\delta}\right)^{\frac{1}{p}}$ and $\left\|f_{1}-f_{2}\right\| \leq \delta$, we obtain

$$
\left\|R\left(f_{1}^{(\alpha)}(\cdot)\right)-R\left(f_{2}^{(\alpha)}(\cdot)\right)\right\| \leq E^{\frac{\alpha}{p}} \delta^{1-\frac{\alpha}{p}}, p>\alpha .
$$

From Lemma 3.1 we see that the derivative defined by (2.7) depends continuously on the input data $f$. Next, we will investigate the difference between the derivatives (2.3) and (2.7) with the same exact $f$.

Lemma 3.2. Let $f^{\alpha}(x)$ and $R\left(f^{\alpha}(x)\right)$ be the derivatives (2.3) and (2.7) with the same exact data $f$, and let $\xi_{\max }=\left(\frac{E}{\delta}\right)^{\frac{1}{p}}, \alpha \leq p<1$. Suppose that $\|f\|_{p} \leq E$.

Then

$$
\left\|f^{\alpha}(\cdot)-R\left(f^{\alpha}(\cdot)\right)\right\| \leq E^{\frac{\alpha}{p}} \delta^{1-\frac{\alpha}{p}} .
$$

Proof. As in Lemma 3.1 we start with the Parseval relation, and using the fact that the derivatives coincide for $\xi \in\left[-\xi_{\max }, \xi_{\max }\right]$ we get 


$$
\begin{aligned}
& \left\|f^{\alpha}(\cdot)-R\left(f^{\alpha}(\cdot)\right)\right\|^{2}=\int_{|\xi|>\xi_{\max }}\left|(i \xi)^{\alpha} \hat{f}(\xi)\right|^{2} \mathrm{~d} \xi \\
& =\int_{|\xi|>\xi_{\max }}|\xi|^{2 \alpha}|\hat{f}(\xi)|^{2} \mathrm{~d} \xi \\
& =\int_{|\xi|>\xi_{\max }} \frac{|\xi|^{2 \alpha}}{\left(1+\xi^{2}\right)^{p}}\left(1+\xi^{2}\right)^{p}|\hat{f}(\xi)|^{2} \mathrm{~d} \xi \\
& \leq \int_{|\xi|>\xi_{\max }}|\xi|^{2(\alpha-p)}\left(1+\xi^{2}\right)^{p}|\hat{f}(\xi)|^{2} \mathrm{~d} \xi \leq \xi_{\max }^{2(\alpha-p)}\|\hat{f}(\cdot)\|_{p}^{2} .
\end{aligned}
$$

Now we use the bound $\|f\|_{p} \leq E$, and as before we have $\xi_{\max }=\left(\frac{E}{\delta}\right)^{\frac{1}{p}}$ which leads to the error bound

$$
\left\|f^{\alpha}(\cdot)-R\left(f^{\alpha}(\cdot)\right)\right\| \leq E^{\frac{\alpha}{p}} \delta^{1-\frac{\alpha}{p}} .
$$

Now we are ready to formulate the main result of this section:

Theorem 3.3. Suppose that $f^{(\alpha)}(x)$ is given by (2.3) with exact data $f$ and that $R\left(f_{\delta}^{(\alpha)}(x)\right)$ is given by (2.7) with measured data $f_{\delta}(x)$. If we have a bound $\|f\|_{p} \leq E$, and the measured function $f_{\delta}(x)$ satisfies $\left\|f-f_{\delta}\right\| \leq \delta$, and if we choose $\xi_{\max }=\left(\frac{E}{\delta}\right)^{\frac{1}{p}}$ where $\alpha \leq p<1$, then we get the error bound

$$
\left\|f^{\alpha}(\cdot)-R\left(f_{\delta}^{(\alpha)}(\cdot)\right)\right\| \leq 2 E^{\frac{\alpha}{p}} \delta^{1-\frac{\alpha}{p}} .
$$

Proof. Let $R\left(f^{\alpha}(x)\right)$ be the derivative defined by (2.7) with exact data $f$. Then by using the triangle inequality and the two previous Lemmas we get

$$
\begin{aligned}
& \left\|f^{\alpha}(\cdot)-R\left(f_{\delta}^{(\alpha)}(\cdot)\right)\right\| \leq\left\|f^{\alpha}(\cdot)-R\left(f^{(\alpha)}(\cdot)\right)\right\| \\
& +\left\|R\left(f^{\alpha}(\cdot)\right)-R\left(f_{\delta}^{(\alpha)}(\cdot)\right)\right\| \leq 2 E^{\frac{\alpha}{p}} \delta^{1-\frac{\alpha}{p}} .
\end{aligned}
$$

From Theorem 3.3, we find that (2.7) is an approximation of the exact derivative $f^{\alpha}(x)$. The approximation error depends continuously on the measurement error.

Remark 3.4. In our application $\|f\|_{p}$ is usually unknown, therefore we have no the exact a priori bound $\mathrm{E}$. However, if we select $\xi_{\max }=C \delta^{p}$, where $C$ is a positive constant, we can also obtain

$$
\left\|f^{\alpha}(\cdot)-R\left(f_{\delta}^{(\alpha)}(\cdot)\right)\right\| \leq C \delta^{1-\frac{1}{p}} \rightarrow 0 \text {, when } \delta \rightarrow 0, \alpha<p,
$$

where the constant $C$ depends on $\alpha, p,\|f\|_{p}$. This choice is helpful in our realistic computation.

\section{Numerical Implement}

Here we discuss how to compute the regularized solution
(2.7) numerically. Given a vector $F_{\delta}$ containing samples from $f_{\delta}$ on an equidistant grid $\left\{x_{j}\right\}_{j=0}^{n-1}$, then the unique trigonometric polynomial interpolating $f_{\delta}$ on the grid can be written

$$
\hat{f}(x)=\frac{1}{\sqrt{2 \pi}} \sum_{j=-\frac{n}{2}}^{\frac{n}{2}-1} \hat{f}_{j} e^{i \xi_{j} x}, \xi_{j}=2 k \pi,
$$

where the sequence $\left\{\hat{f}_{j}\right\}_{j=-\frac{n}{2}}^{\frac{n}{2}-1}$ are the discrete Fourier coefficients, and we have assumed that $n$ is even. The discrete Fourier coefficients can be computed by taking the FFT of the vector $F_{\delta}$. Thus we can approximate the derivative operator (2.7) as follow:

$$
R\left(F_{\delta}^{(\alpha)}\right)=L^{H} \Lambda^{\alpha} L F_{\delta},
$$

where $L$ is the Fourier matrix [9], and $\Lambda$ is a diagonal matrix corresponding to differentiation of trigonometric interpolant, but where the frequency components with $\left|\xi_{j}\right|>\xi_{\max }$ are explicitly set to zero. Thus the diagonal elements of $\Lambda$ are

$$
(\Lambda)_{i j}= \begin{cases}i \xi_{j}, & \left|\xi_{j}\right| \leq \xi_{\max }, \\ 0, & \left|\xi_{j}\right|>\xi_{\max },\end{cases}
$$

where $\xi_{j}$ are defined as in (4.1). The product of $L$ and a vector can be computed using the Fast Fourier Transform (FFT) which leads to an efficient way to compute the derivative (2.7). When using the FFT algorithm we implicitly assume that the vector $F_{\delta}$ represents a periodic function. This is not realistic in our application, and thus we need to modify the algorithm. A discussion on how to make the function "periodic" can be found in [12].

\section{Numerical Examples}

For verifying the effect of the proposed algorithm, a smooth function and a non-smooth function will be tested in various cases. In the numerical experiment, we always fix $0 \leq x \leq 1$. For an exact data function $f(x)$, its discrete noisy version is

$$
f_{\delta}=f+\varepsilon \operatorname{randn}(\operatorname{size}(f)),
$$

where

$$
\begin{aligned}
& f=\left(f\left(x_{1}\right), \cdots f\left(x_{N}\right)\right), x_{j}=\frac{j-1}{N-1}, j=1,2, \cdots, N, \\
& \delta=\left\|f_{\delta}-f\right\|_{l^{2}}=\sqrt{\frac{1}{N} \sum_{j=1}^{N}\left(f\left(x_{j}\right)-f_{\delta}\left(x_{j}\right)\right)^{2}} .
\end{aligned}
$$

The function " randn(.)" generates arrays of random numbers whose elements are normally distributed with 
mean 0 , variance $\sigma^{2}=1$, and standard deviation $\sigma=1$, " $\operatorname{randn}(\operatorname{size}(f))$ "returns an array of random entries that is the same size as $f$. In our computations, we always take $N=4097$ (If we take $N=100,513,1025, \cdots$, we can also obtain a satisfactory result.)The derivative errors are measured by the weighted $L^{2}$-norms defined as follows:

$$
E\left(R\left(f_{\delta}^{(\alpha)}\right)\right)=\sqrt{\frac{1}{N} \sum_{j=1}^{N}\left(f^{(\alpha)}\left(x_{j}\right)-R\left(f_{\delta}^{(\alpha)}\left(x_{j}\right)\right)\right)^{2}} .
$$

The regularization parameter $\xi_{\max }$ was selected according to Remark 3.4, i.e., $\xi_{\max }=c \delta^{-\frac{1}{p}}$. The error estimates of Section 3 contain norms in $L^{2}(R)$, and the numerical experiments are performed with a finite interval. However, the method for selecting $\xi_{\max }$ works well,also in the case where the norms are computed for a finite interval.

\section{Acknowledgements}

The author wants to express his thanks to the referee for many valuable comments. This work is supported by Educational Commission of Hubei Province of China (Q20102804,T201009).

\section{References}

[1] J. Baumeister, "Stable Solution of Inverse Problems," F. Vieweg and Sohn, Braunschweig, 1987.

[2] R. Gorenflo and S. Vessela, "Abel Integral Equations," Springer-Verlag, Berlin, Heidelberg, New York, 1991.
[3] A. K. Louis, "Inverse und Schlecht Gesteellte Problem," Teubner Verlag, Wiesbaden, 1989.

[4] D. A. Murio, "Automatic Numerical Differentiation by Discrete Molification," Computers and Mathematics with Applications, Vol. 13, No. 4, 1987, pp. 381-386. doi:10.1016/0898-1221(87)90006-X

[5] D. A. Murio and L. Guo, "Discrete Stability Analysis of Molification Method for Numerical Differentiation," Journal of Computational Applied Mathematics, Vol. 19, No. 6, 1990, pp. 15-25.

[6] I. Daubechies, "Ten Lectures on Wavelets (CBMS-NSF Regional Conference Series in Applied Mathematics)," SIAM: Society for Industrial and Applied Mathematics, Philadelphia, 1992.

[7] J. N. Lyness, "Has Numerical Differentiation a Future?" Utilitas Mathematics, Winnipeg, 1978.

[8] J. Oliver, "An Algorithm for Numerical Differentiation of a Funcion of One Real Variable," Journal of Computational Applied Mathematics, Vol. 6, No. 2, 1980, pp. 145160. doi:10.1016/0771-050X(80)90008-X

[9] T. Strom and J. N. Lyness, "On Numerical Differentiation,” BIT Numerical Mathematics, Vol. 15, No. 3, 1975, pp. 314-322. doi:10.1007/BF01933664

[10] D. N. Hao, "A Molification Method for Ill-Posed Problems," Numerische Mathematik, Vol. 68. No. 4, 1994, pp. 469-506. doi:10.1007/s002110050073

[11] D. A. Murio, C. E. Mejia and S. Zhan, "Discrete Molification and Automatic Numerical Differentiation," Computers and Mathematics Application, Vol. 35, No. 5, 1998, pp. 1-16. doi:10.1016/S0898-1221(98)00001-7

[12] L. Elden, F. Berntsson and T. Reginska, "Wavelet and Fourier Methods For Solving the Sideways Heat Equation," SIAM Journal on Scientific Computing, Vol. 21, No. 6, 2000, pp. 2187-2205. 